\title{
Avaliação da acuidade visual e da pressão intraocular no tratamento do edema macular diabético com triancinolona intravítrea
}

\author{
Study of visual acuity and intraocular pressure in the treatment of \\ macular diabetic edema with intravitreous triamcinolone
}

\author{
Marcussi Palata Rezende ${ }^{1}$ \\ Alana Ferreira Gomes Dias ${ }^{2}$ \\ Akiyoshi Oshima $^{3}$ \\ Eric Pinheiro de Andrade ${ }^{4}$ \\ Pedro Durães Serracarbassa ${ }^{5}$
}

Trabalho realizado no Setor de Retina e Vítreo do Departamento de Oftalmologia do Hospital do Servidor Público Estadual de São Paulo - HSPE/IAMSPE - São Paulo (SP) - Brasil.

${ }^{1}$ Pós-graduando nível mestrado do Hospital do Servidor Público Estadual de São Paulo - HSPE - São Paulo (SP) - Brasil.

${ }^{2}$ Pós-graduanda nível mestrado do HSPE - São Paulo (SP) - Brasil.

${ }^{3}$ Doutor em Oftalmologia pela Universidade Federal de São Paulo - UNIFESP - São Paulo (SP) - Brasil. Chefe do Serviço de Retina e Vítreo do HSPE São Paulo (SP) Brasil.

${ }^{4}$ Chefe do Serviço de Neuroftalmologia do HSPE - São Paulo (SP) - Brasil.

${ }^{5}$ Doutor em Medicina pela Universidade de São Paulo USP - São Paulo (SP) - Brasil; Médico Assistente do Serviço de Retina e Vítreo do HSPE São Paulo (SP) Brasil.

Endereço para correspondência: Marcussi Palata Rezende. Secretaria de Oftalmologia do HSPE/IAMSPE. Rua Borges Lagoa, 1.755 - $3^{\circ}$ andar - sala 305 - São Paulo (SP) CEP 04038-034

E-mail: marcussipr@hotmail.com

Recebido para publicação em 06.11.2008

Última versão recebida em 25.01.2010

Aprovação em 09.02.2010

Nota Editorial: Depois de concluída a análise do artigo sob sigilo editorial e com a anuência dos Drs. João Borges Fortes Filho e João J. Nassaralla Jr. sobre a divulgação de seus nomes como revisores, agradecemos suas participações neste processo.

\section{RESUMO}

Objetivos: Avaliar os efeitos do acetato de triancinolona intravítreo em pacientes com edema macular diabético difuso na acuidade visual e pressão intraocular. Relatar os possíveis efeitos adversos e analisar a possível relação da idade dos pacientes com as variações da acuidade visual e pressão intraocular. Métodos: $O$ ensaio clínico controlado incluiu 14 pacientes ( 28 olhos), sendo que 14 olhos receberam injeção de $4 \mathrm{mg}$ de acetato de triancinolona intravítreo para o tratamento de edema macular diabético difuso. $\mathrm{O}$ grupo tratado foi comparado a um grupo controle de 14 olhos sem edema macular diabético difuso. $\mathrm{O}$ tempo de seguimento foi de três meses. Resultados: Os picos de pressão intraocular $>21 \mathrm{mmHg}$ ocorreram em 28,57\%, com diferença significante entre a pressão intraocular do grupo tratado com o grupo controle na primeira semana após o tratamento. A acuidade visual mostrou uma significativa melhora quando comparada com o grupo controle desde o segundo dia após o tratamento. Não houve associação entre a idade com as variações da acuidade visual e a pressão intraocular. Conclusão: $\mathrm{O}$ acetato de triancinolona intravítreo mostrou-se ser eficiente para melhorar a acuidade visual em pacientes com edema macular diabético difuso, nos primeiros três meses de tratamento. A incidência de hipertensão intraocular foi de $28,57 \%$, podendo ser caracterizada como de fácil controle.

Descritores: Acetato de triancinolona/uso terapêutico; Retinopatia diabética; Edema macular/efeito de drogas; Pressão intraocular; Hipertensão ocular; Acuidade visual

\section{INTRODUÇÃO}

A retinopatia diabética $(\mathrm{RD})$ é caracterizada por microangiopatia retiniana secundária à quebra da barreira hematorretiniana que determina alterações típicas com aspectos tanto de oclusão quanto de extravasamento microvascular e com a consequente formação de "shunts" arteriovenosos e neovascularização ${ }^{(1-2)}$.

$\mathrm{Na}$ fisiopatologia do edema macular diabético (EMD) ocorre a perda de pericitos, a formação de microaneurismas, o espessamento da membrana basal, a oclusão focal dos capilares e a quebra na barreira retiniana interna, ocasionando aumento da permeabilidade vascular ${ }^{(3)}$. Nos pacientes com $\mathrm{RD}$ a causa mais comum de baixa de acuidade visual classifica-se em: a) edema macular diabético focal, em que se observa edema retiniano focal, 
proveniente dos microaneurismas, com presença comum dos exsudatos duros; $b$ ) edema macular diabético difuso (EMDD), que representa uma falência generalizada da barreira hematorretiniana interna, havendo vazamento difuso de microaneurismas, capilares e anomalias microvasculares intrarretinianas (IRMA) $)^{(4-6)}$. Seus principais fatores de risco são: hiperglicemia, hipertensão arterial sistêmica, hiperlipedemia, duração do diabetes, gravidez e intervenção oftalmológica como panfotocoagulação retiniana e facectomia ${ }^{(3)}$.

Como a literatura mostra que o laser convencional não resulta em melhora significativa em pacientes com edema macular grave, novos métodos terapêuticos têm sido emprega$\operatorname{dos}^{(3)}$. O acetato de triancinolona (AT) tem sido usado como tratamento primário ou adjunto para o EMDD, mostrando resultados promissores com poucas complicações ${ }^{(3,7-9)}$.

Os objetivos deste trabalho são: a) avaliar a pressão intraocular (PIO) e a acuidade visual (AV) de pacientes normotensos oculares com EMDD submetidos à injeção de acetato de triancinolona intravítreo (ATIV), por um período de três meses; b) relatar as possíveis complicações do uso desse medicamento e c) analisar a correlação da idade com as variações da acuidade visual e pressão intraocular durante o tratamento.

\section{MÉTODOS}

Foi realizado um ensaio clínico controlado no Setor de Retina do Departamento de Oftalmologia do Hospital do Servidor Público Estadual de São Paulo, no período de fevereiro de 2007 a julho de 2007. O tempo de seguimento foi estabelecido em três meses. No estudo se incluíram 28 olhos de 14 pacientes, em que apenas um dos olhos apresentavam EMDD. Os olhos foram divididos em dois grupos: 1) grupo tratado: formados por pacientes que apresentavam EMDD e receberam tratamento com ATIV; 2) grupo-controle: formado por pacientes cujo olho contralateral não apresentava EMDD.

O protocolo de pesquisa foi aprovado pelo Comitê de Ética do Hospital do Servidor Público Estadual de São Paulo, e todos os pacientes assinaram termo de consentimento livre esclarecido.

Os critérios de inclusão do paciente foram: a) ser portador de EMDD, com ou sem tratamento prévio realizado com fotocoagulação a laser; b) apresentar diminuição da acuidade visual; c) ter PIO normal; d) mostrar-se colaborativo; e) assinar termo de consentimento livre esclarecido; f) não ser portador de nenhuma outra doença retiniana.

Os critérios de exclusão foram: a) ser portador de edema macular de outra etiologia; b) apresentar EMDD em ambos os olhos c) ter recebido previamente injeção de ATIV; d) possuir olho único; e) ser portador de glaucoma ou hipertensão intraocular (PIO maior que $21 \mathrm{mmHg}$ ); f) ter sido submetido a uma cirurgia vitreorretiniana; g) apresentar lesão retiniana predisponente para descolamento de retina.

A injeção de ATIV foi realizada no centro cirúrgico seguindo o seguinte protocolo: instilou-se colírio anestésico e iodo- povidona a 5\% no saco conjuntival; colocou-se bolsa de mercúrio para descompressão ocular; após dez minutos, os cílios foram isolados com campo cirúrgico de plástico e blefarostato, para então ser injetado AT sem conservante $0,1 \mathrm{ml}(4 \mathrm{mg})$ intravítreo, via pars plana, com agulha 25 gauge a $4,00 \mathrm{~mm}$ do limbo em pacientes fácicos e a $3,50 \mathrm{~mm}$ do limbo em pseudofácicos. Caso se constatasse aumento da PIO, pela tonometria oculodigital, ou diminuição da perfusão retiniana, observada através da oftalmoscopia binocular indireta, a paracentese seria indicada. Após o procedimento foi utilizado colírio de antibiótico (tobramicina $0,3 \%)$ de quatro em quatro horas durante cinco dias, para prevenção de infecção ocular. Pacientes com hipertensão intraocular (HIO) receberam tratamento com colírio hipotensor.

Os pacientes responderam um questionário em que constavam perguntas sobre sexo, idade, antecedentes pessoais, uso de medicação, tratamento ocular prévio, sintomas (sensação de corpo estranho) e avaliação subjetiva da acuidade visual (AV) durante o decorrer do seguimento. Foram realizados cinco consultas nos três meses de acompanhamento. $\mathrm{Na}$ primeira consulta (dia 1), procedeu-se ao exame momentos antes de o paciente receber o tratamento ("baseline"); a segunda consulta (dia 2), foi realizada no dia seguinte ao tratamento; a terceira consulta ( $1^{\underline{a}}$ semana), ocorreu uma semana após o tratamento; a quarta consulta ( $1^{\circ}$ mês), foi feita um mês após o tratamento, e a quinta consulta ( 3 meses), se deu três meses após o tratamento. Em todas as consultas, os pacientes foram submetidos a exames de AV corrigida, biomicroscopia, tonometria de aplanação, fundoscopia e retinografia.

A AV foi convertida do padrão da tabela de Snellen para $\log$ MAR. Para comparar se há alteração nas medidas de PIO e AV entre o grupo tratado e o controle ao longo do tratamento foram realizadas análises de variâncias. Após as análises, os valores foram comparados entre os grupos em cada momento, bem como nos mesmo grupo, nos diversos momentos, com uso de comparações múltiplas de Tukey. Verificou-se a relação entre a variação sofrida na $\mathrm{AV}$ e na PIO com a idade dos pacientes com uso de correlações de Pearson. Os testes foram realizados com significância de $5 \%(\mathrm{p}<0,05)$. O programa utilizado foi SPSS 15.0.

\section{RESULTADOS}

A média de idade dos pacientes variou de 53 a 81 (média de $65,86 \pm 10,95$ anos), sendo que a grande maioria dos pacientes $(92,85 \%)$ já havia realizado tratamento prévio com fotocoagulação a laser para tratamento do EMDD.

Como se pode observar na tabela $1,79 \%$ dos pacientes eram do sexo feminino, tendo o olho direito $64 \%$ da medição recebida. A maioria dos pacientes relatou melhora da $\mathrm{AV}$ a partir do segundo dia e sensação de corpo estranho apenas até a primeira semana.

Em todos os pacientes se colocou bolsa de mercúrio no olho que iria receber a injeção de ATIV e em nenhum deles foi necessário realizar paracentese. Durante o seguimento não foi 
observado progressão da catarata, endoftalmite infecciosa ou não, descolamento de retina e catarata traumática.

A principal complicação observada foi HIO, sendo o maior pico pressórico de $28 \mathrm{mmHg}$. Quatro pacientes $(28,57 \%)$ apresentaram PIO maior que $21 \mathrm{mmHg}$, na primeira semana após a injeção de ATIV. Todos receberam colírio hipotensor (maleato de timolol 0,5\%, uma gota de 12 em 12 horas) até o primeiro mês após o procedimento, com retorno pressórico inferior a $21 \mathrm{mmHg}$, quando então foi retirado o medicamento. Nenhum dos pacientes foi submetido à cirurgia filtrante.

\begin{tabular}{|c|c|c|}
\hline Variável & Frequência & Porcentual (\%) \\
\hline \multicolumn{3}{|l|}{ Sexo } \\
\hline Feminino & 11 & 78,6 \\
\hline Masculino & 3 & 21,4 \\
\hline \multicolumn{3}{|l|}{ Olho } \\
\hline Direito & 9 & 64,3 \\
\hline Esquerdo & 5 & 35,7 \\
\hline Necessidade de colírio hipotensor & 4 & 28,5 \\
\hline \multicolumn{3}{|l|}{ Melhora da AV } \\
\hline Dia 2 & 12 & 85,7 \\
\hline 1a semana & 11 & 78,6 \\
\hline $1^{\circ}$ mês & 9 & 64,3 \\
\hline 3 meses & 12 & 85,7 \\
\hline \multicolumn{3}{|l|}{ Sensação de corpo estranho } \\
\hline Dia 2 & 4 & 28,6 \\
\hline 1a semana & 2 & 14,3 \\
\hline $1^{\circ}$ mês & 0 & 0,0 \\
\hline 3 meses & 0 & 0,0 \\
\hline \multicolumn{3}{|c|}{$\begin{array}{l}\text { Dia } 2 \text { = primeiro dia após receber o tratamento de acetato de triancinolona intra- } \\
\text { vítreo; } 1^{\underline{a}} \text { semana }=\text { uma semana após receber o tratamento de ATIV; } 1^{\circ} \text { mês= } \\
1 \text { mês após receber o tratamento de ATIV; } 3 \text { meses= três meses após receber } \\
\text { o tratamento de ATIV }\end{array}$} \\
\hline
\end{tabular}

A tabela 2 mostra as médias e desvios-padrões da PIO por olho e o tempo de acompanhamento. A média com maior valor da PIO ocorre na primeira semana $(19,21 \pm 4,48)$, enquanto no grupo controle não se observou o mesmo.

A PIO média no grupo tratado foi superior na primeira semana com relação ao dia $1(\mathrm{p}=0,002)$ e também é superior ao grupo-controle na primeira semana $(\mathrm{p}=0,006)$. Assim, ao compararmos as médias da PIO do grupo tratado ao longo do estudo com a PIO inicial, observa-se uma diferença significativa somente na primeira semana após o tratamento. Quando se comparou a PIO do grupo tratado com a do grupo-controle, houve diferença somente na primeira semana após o tratamento. Para o restante dos momentos, em que foram feitas as aferições não se observou diferença (Tabela 3 ).

O gráfico 1 mostra que a PIO média no grupo tratado aumentou até a primeira semana, havendo então diminuição e aproximando-se dos valores do grupo-controle.

A média da AV antes do procedimento foi de 1,55 $\pm 0,91$ para o grupo tratado e de $0,96 \pm 0,67$ para o grupo-controle. Após três meses de tratamento, observou-se uma média da AV de $0,99 \pm 0,47$ no grupo tratado e de $0,86 \pm 0,60$ no grupo não tratado. A tabela 2 mostra as médias da AV no grupo tratado e no grupo-controle ao longo do estudo.

A AV no grupo tratado foi em média estatisticamente melhor que o dia 1 já na primeira semana de tratamento e continuou a melhorar até três meses de tratamento $(\mathrm{p}<0,05)$. No dia 2, a $\mathrm{AV}$ dos grupos tratado e não tratado se iguala em média ( $>0,05)$, sendo estatisticamente pior no olho tratado apenas no dia $1(\mathrm{p}<0,001)$. Assim ao comparar as médias da AV do grupo tratado ao longo do estudo com a AV inicial, observa-se uma diferença estatisticamente significativa da $\mathrm{AV}$ na primeira semana, com um mês e com três meses. Quando se comparou a AV do grupo tratado com o grupo-controle, houve diferença entre eles no dia 1 , com diminuição do valor de $\mathrm{p}$ entre os grupos nos demais dias de acompanhamento (Tabela 3). O gráfico 2 mostra que há uma significante diferença entre os valores da AV entre o grupo tratado e o controle antes de re-

Tabela 2 . Descrição da média e desvio-padrão da PIO e AV no grupo tratado e grupo-controle nos diversos momentos do acompanhamento ao longo de três meses

\begin{tabular}{|c|c|c|c|c|c|}
\hline \multirow[b]{2}{*}{ Variável } & \multirow[b]{2}{*}{ Momento } & \multicolumn{2}{|c|}{ G T } & \multicolumn{2}{|c|}{ GC } \\
\hline & & Média & DP & Média & DP \\
\hline \multirow{5}{*}{$\mathrm{PIO}$} & Dia 1 & 15,43 & 2,14 & 15,64 & 1,86 \\
\hline & Dia 2 & 17,00 & 2,96 & 15,50 & 2,35 \\
\hline & 1 semana & 19,21 & 4,48 & 15,79 & 2,94 \\
\hline & $1^{\circ}$ mês & 15,93 & 2,76 & 15,14 & 2,66 \\
\hline & 3 meses & 15,64 & 2,21 & 15,00 & 2,08 \\
\hline \multirow{5}{*}{ AV } & Dia 1 & 1,55 & 0,91 & 0,96 & 0,67 \\
\hline & Dia 2 & 1,19 & 0,55 & 0,94 & 0,70 \\
\hline & 1 semana & 1,09 & 0,54 & 0,88 & 0,62 \\
\hline & $1^{\circ}$ mês & 1,02 & 0,48 & 0,87 & 0,60 \\
\hline & 3 meses & 0,99 & 0,47 & 0,86 & 0,60 \\
\hline
\end{tabular}




\begin{tabular}{|c|c|c|c|}
\hline Variável & Fator & Comparação & p \\
\hline \multirow{13}{*}{ PIO } & \multirow[t]{4}{*}{$\mathrm{GT}$} & Dia 1 - Dia 2 & 0,691 \\
\hline & & Dia $1-1^{\underline{a}}$ semana & 0,002 \\
\hline & & Dia 1 - $1^{\circ}$ mês & $>0,999$ \\
\hline & & Dia 1 - 3 meses & $>0,999$ \\
\hline & \multirow[t]{4}{*}{$\overline{\mathrm{GC}}$} & Dia 1 - Dia 2 & $>0,999$ \\
\hline & & Dia 1 - 1a semana & $>0,999$ \\
\hline & & Dia 1 - $1^{\circ}$ mês & $>0,999$ \\
\hline & & Dia 1 - 3 meses & 0,999 \\
\hline & Dia 1 & $\mathrm{GT}-\mathrm{GC}$ & $>0,999$ \\
\hline & Dia 2 & $\mathrm{GT}-\mathrm{GC}$ & 0,744 \\
\hline & $1^{a}$ semana & $\mathrm{GT}-\mathrm{GC}$ & 0,006 \\
\hline & $1^{\circ}$ mês & $\mathrm{GT}-\mathrm{GC}$ & 0,995 \\
\hline & 3 meses & $\mathrm{GT}-\mathrm{GC}$ & 0,999 \\
\hline \multirow{13}{*}{ AV } & \multirow[t]{4}{*}{$\mathrm{GT}$} & Dia 1 - Dia 2 & 0,072 \\
\hline & & Dia $1-1^{a}$ semana & 0,007 \\
\hline & & Dia 1 - $1^{\circ}$ mês & 0,001 \\
\hline & & Dia 1 - 3 meses & $<0,001$ \\
\hline & \multirow[t]{4}{*}{$\mathrm{GC}$} & Dia 1 - Dia 2 & $>0,999$ \\
\hline & & Dia $1-1^{a}$ semana & $>0,999$ \\
\hline & & Dia $1-1^{\circ}$ mês & 0,998 \\
\hline & & Dia $1-3$ meses & 0,996 \\
\hline & $\overline{\text { Dia } 1}$ & GT - GC & $<0,001$ \\
\hline & Dia 2 & $\mathrm{GT}-\mathrm{GC}$ & 0,472 \\
\hline & $1^{a}$ semana & $\mathrm{GT}-\mathrm{GC}$ & 0,722 \\
\hline & $1^{\circ}$ mês & $\mathrm{GT}-\mathrm{GC}$ & 0,947 \\
\hline & 3 meses & $G T-G C$ & 0,972 \\
\hline \multicolumn{4}{|c|}{$\begin{array}{l}\mathrm{PIO}=\text { pressão intraocular; } \mathrm{AV}=\text { acuidade visual; } \mathrm{GT}=\text { grupo tratado; } \mathrm{GC}=\text { grupo- } \\
\text { controle; Dia } 1=\text { dia em que o paciente recebe o tratamento de ATIV; Dia } 2= \\
\text { um dia após receber o tratamento de ATIV; } 1 \text { a semana= uma semana após } \\
\text { receber o tratamento de ATIV; } 1^{\circ} \text { mês= primeiro mês após receber o tratamento } \\
\text { de ATIV; } 3 \text { meses= três meses após receber o tratamento de ATIV }\end{array}$} \\
\hline
\end{tabular}

ceber o tratamento, diferença esta que vai diminuindo ao longo do estudo. Assim se observou uma melhora da AV no grupo tratado, que tende a se aproximar dos valores da AV do grupo-controle já na primeira semana de tratamento.

Os gráficos 1 e 2 mostram que o comportamento da $\mathrm{PIO}$ e da AV (ANOVA) não são os mesmos ao longo do acompanhamento para os dois grupos $(p=0,043$ e $p=0,040$, respectivamente).

No presente estudo não existiu correlação estatisticamente significativa entre a idade e as variações sofridas na PIO e AV ( $p>0,05)$, como mostra a tabela 4 .

\section{DISCUSSÃO}

Os estudos em animais têm mostrado que a injeção de ATIV estabiliza a barreira hematorretiniana e diminui a fisiopatologia da retinopatia proliferativa ${ }^{(7,10)}$. O AT age diminuindo os mediadores da inflamação, como as prostaglandinas e as interleucinas, responsáveis pela gênese do edema macu$\operatorname{lar}^{(3,7,11)}$. Elas bloqueiam a produção do fator de crescimento endotelial, responsável pela neovascularização retiniana e pelo aumento da permeabilidade vascular ${ }^{(3,7,12)}$.

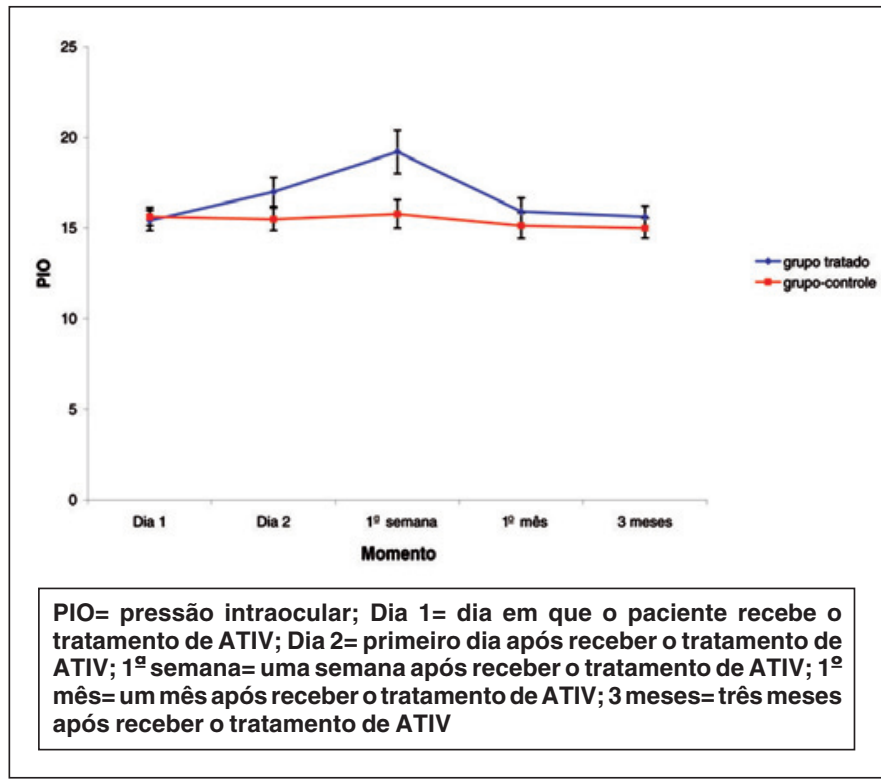

Gráfico 1 - Variação da média da PIO durante o seguimento de três meses nos grupo tratado e no grupo-controle

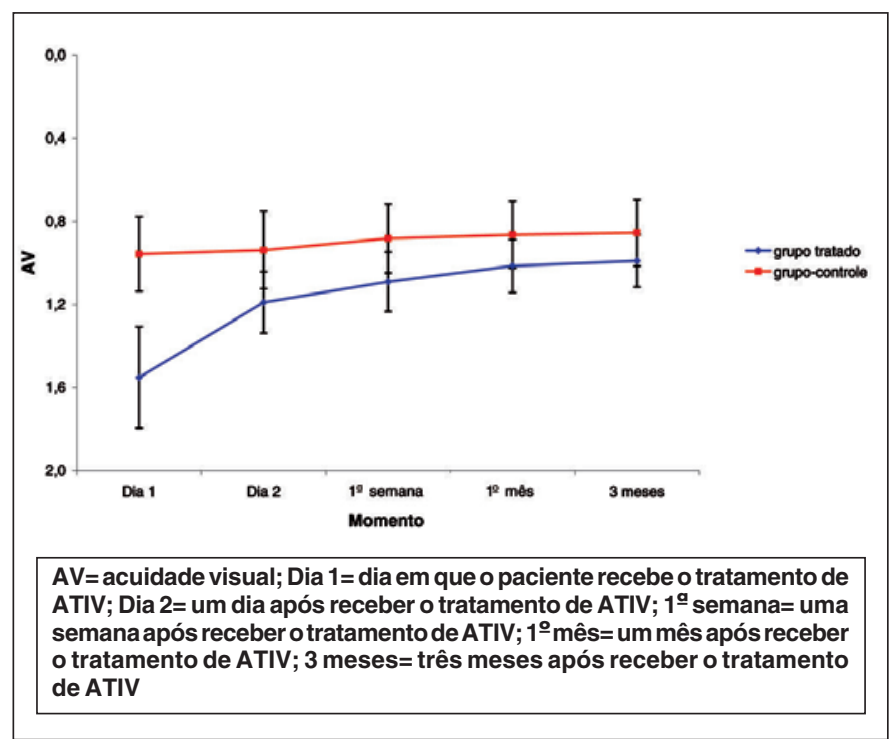

Gráfico 2 - Variação da média da AV durante o seguimento de três meses nos grupo tratado e no grupo-controle

Tabela 4. Correlação entre a idade e as variações (finais-iniciais) sofridas na pressão intraocular e na acuidade visual nos olhos submetidos ao tratamento e nos olhos não submetidos ao tratamento ao longo do estudo

$\begin{array}{lc}\text { Variáveis } & \mathbf{p} \\ \text { Idade vs Variação da PIO GT } & 0,449 \\ \text { Idade vs Variação da PIO GC } & 0,796 \\ \text { Idade vs Variação da AV GT } & 0,244 \\ \text { Idade vs Variação da AV GC } & 0,325 \\ \text { PIO GT= pressão intraocular no grupo tratado; PIO GC= pressão intraocular no } \\ \begin{array}{l}\text { grupo-controle; AV GT= acuidade visual no grupo tratado; AV GC= acuidade } \\ \text { visual grupo-controle }\end{array}\end{array}$


O AT tem sido usado como terapia adjunta para tratamento de edema macular diabético, de degeneração macular relacionada à idade, de retinopatia diabética proliferativa, de oclusão de veia central da retina e de uveíte com edema macular cistoide ${ }^{(3,12-15)}$. A segurança da administração intravítrea vem sendo confirmada em trabalhos anteriores em animais e em "trials" em humanos ${ }^{(3,16)}$. A biodisponibilidade do AT, quando usada intravítrea, é maior quando comprada com a administração subtenoniana, não sendo tóxico para a retina em doses de $1 \mathrm{a} 4 \mathrm{mg}$ em olhos vitrectomizados e não vitrectomizados $^{(3,17-18)}$.

Observou-se HIO em 4 pacientes (28,57\%), um resultado que condiz com os encontrados na literatura médica $(20-64 \%)^{(19)}$. Essa variação ocorre em decorrência da dose do corticosteróide, do critério para hipertensão, do tempo de seguimento e da porcentagem de retratamento ${ }^{(19)}$. Com o aumento da dose utilizada, há uma maior incidência de hipertensão ocular ${ }^{(8,12,19)}$. No presente estudo, foram utilizados $4 \mathrm{mg}$ e apenas uma injeção; quando se compara com trabalhos que fizeram uso da mesma concentração da droga encontram-se as seguintes incidências de HIO: $39 \%^{(8)} ; 27,5 \%^{(19)} ; 23 \%^{(20)} ; 53,2 \%^{(21)}$ e $44 \%^{(22)}$. Todos os casos de HIO ocorreram dentro do primeiro mês de tratamento. Um trabalho mostrou que $82,6 \%$ dos casos de $\mathrm{HIO}$ foram registrados nos primeiros três meses, sendo apenas $20,1 \%$ no primeiro mês ${ }^{(19)}$; um outro descreveu que todos os casos se manifestaram nos primeiros três meses ${ }^{(20)}$. A HIO relatada no presente estudo foi de fácil controle com o uso de um único colírio hipotensor (maleato de timolol 0,5\%), como preconiza a literatura ${ }^{(8,19-21,23)}$. A causa da hipertensão se deve principalmente a uma diminuição da drenagem do humor aquoso em função de um remodelamento da malha trabecular e também a uma obstrução do trabeculado pelos cristais $\mathrm{AT}^{(14,19)}$.

Os resultados encontrados neste estudo são semelhantes aos encontrados em trabalhos prévios, confirmando que ATIV pode melhorar a AV em pacientes com edema macular diabético difuso devido à retinopatia diabética ${ }^{(1)}$. Trabalhos mostram que a AV tende a melhorar até os três primeiros meses de tratamento $^{(3,14,17)}$ e começar a regredir do terceiro até o sexto mês de tratamento ${ }^{(20-21)}$. Demonstrou-se neste estudo que a melhora da AV é observada na primeira semana após o tratamento, podendo chegar a valores próximos do grupo-controle até o terceiro mês após o tratamento. A AV não mudou significativamente no grupo-controle durante o seguimento, assim como também em trabalhos já publicados ${ }^{(3,17)}$.

Não se observou progressão da catarata, assim como em outros trabalhos ${ }^{(20-21,23)}$. Isto pode ser explicado pelo pequeno tempo de seguimento deste estudo (três meses) para o desenvolvimento de catarata. Alguns autores em um trabalho de aproximadamente nove meses de duração, relataram uma progressão da catarata de $6,6 \%{ }^{(24)}$. Não houve necessidade de realizar paracentese em nenhum dos pacientes, visto que, ao colocar a bolsa de mercúrio para descompressão ocular, esta reduz a tonicidade do olho ${ }^{(25)}$, além de ser um risco adicional para o desenvolvimento de endoftalmite ${ }^{(26)}$.
Não se observou endoftalmite com cultura positiva, assim como em outros trabalhos ${ }^{(17,19-21)}$, embora na literatura possa encontrar-se uma incidência de $0,87 \%{ }^{(15)}$.

Em nenhum caso foi preciso realizar cirurgia filtrante ${ }^{(20)}$. Há trabalhos que mostram uma incidência de $0,094 \%{ }^{(12)}$, $5,88 \%{ }^{(22)}$, sendo o valor médio encontrado na literatura de aproximadamente $1 \%{ }^{(19)}$.

Não existiu correlação estatisticamente significativa entre a idade e as variações sofridas na PIO e AV no decorrer do trabalho, assim como verificado na literatura ${ }^{(19)}$, embora um estudo tenha mostrado uma associação maior para hipertensão em pacientes jovens ${ }^{(21)}$.

\section{CONCLUSÃO}

Houve melhora significativa da AV nos pacientes tratados quando comparados ao grupo-controle.

A PIO mostrou um aumento significativo, principalmente na primeira semana após o tratamento, sendo, porém, de fácil controle e tendo retornado para valores normais a partir do primeiro mês após a injeção de ATIV.

O tratamento realizado mostrou ser seguro pela ausência de complicações, tais como: progressão da catarata, endoftalmite infecciosa ou asséptica, descolamento de retina, catarata traumática, glaucoma de difícil controle e necessidade de cirurgia filtrante.

A idade dos pacientes não mostrou relação com as variações da AV e PIO ocorridas no decorrer deste trabalho.

\section{ABSTRACT}

Purpose: To evaluate the effect of intravitreal triamcinolone acetonide in patients with diffuse diabetic macular edema on the visual acuity and intraocular pressure. To report the potential adverse events and to analyze the potential relationship between age and visual acuity and intraocular pressure variability. Methods: This clinical controlled study included 14 patients (28 eyes), 14 of the eyes received an intravitreal injection of $4 \mathrm{mg}$ triamcinolone acetonide for the treatment of diabetic macular edema. The study group was compared to a control group of 14 eyes, without diabetic macular edema. The follow-up period was of 3 months. Results: Pressure spikes $>21 \mathrm{mmHg}$ occurred in $28.7 \%$ of eyes, with a significant difference of intraocular pressure between the study group and the control group in the first week after treatment. Visual acuity showed a significant improvement when compared with the control group since the second day after the treatment. There was no association between age and variability of visual acuity and intraocular pressure. Conclusion: Intravitreal triamcinolone acetonide showed to be effective for improving visual acuity in patients with diabetic macular edema, in the first three months of treatment. The incidence of intraocular hypertension was $28.7 \%$, easily manageable. 
Keywords: Triamcinolone acetonide/therapeutic use; Diabetic retinopathy; Macular edema/drug effects; Intraocular pressure; Ocular hypertension; Visual acuity

\section{REFERÊNCIAS}

1. Esteves J, Laranjeira AF, Roggia MF, Dalpizol M, Scocco C, Kramer CK, et al. Fatores de risco para retinopatia diabética. Arq Bras Endocrinol Metab. 2008;52(3):431-41.

2. Cogan DG, Toussaint D, Kuwabara T. Retinal vascular patterns. IV. Diabetic retinopathy. Arch Ophthalmol. 1961;66:366-78.

3. Chieh JJ, Roth DB, Liu M, Belmont J, Nelson M, Regillo C, et al. Intravitreal triamcinolone acetonide for diabetic macular edema. Retina. 2005;25(7):828-34.

4. Schachat AP, Murphy RP, editors. Medical retina. In: Ryan SJ, editor. Retina. $4^{\text {th }}$ ed. St. Louis: Mosby; 2006. v.2, p.1241-323.

5. Khairallah M, Zeghidi H, Ladjimi A, Yahia SB, Attia S, Zaouali S, et al. Primary intravitreal triamcinolone acetonide for diabetic massive macular hard exsudates. Retina. 2005;25(7):835-9.

6. Photocoagulation for diabetic macular edema. Early Treatment of Diabetic Retinopathy Study report number 1. Early Treatment Diabetic Retinopathy Study research group. Arch Ophthalmol. 1985;103(12):1796-806.

7. Zein WM, Noureddin BN, Jurdi FA, Schakal A, Bashshur ZF. Panretinal photocoagulation and intravitreal triamcinolone acetonide for the management of proliferative diabetic retinopathy with macular edema. Retina. 2006;26(2): $137-42$.

8. Lam DS, Chan Ck, Mohamed S, Lai TY, LI KK, Li PS, et al. A Prospective randomised trial of different doses of intravitreal triamcinolone for diabetic macular edema. Br J Ophthalmol. 2007;91(2):199-203.

9. Enaida H, Hata Y, Ueno A, Nakamura T, Hisatomi T, Miyazaki M, et al. Possible benefits of triamcinolone-assisted pars plana vitrectomy for retinal diseases. Retina. 2003;23(6):764-70.

10. Wilson CA, Berkowitz B, Sato Y, Ando N, Hand JT, de Juan E. Treatment with intravitreal steroid reduces blood-retinal barrier breakdown due to retinal photocoagulation. Arch Ophthalmol. 1992;110(8):1155-9.

11. Funatsu H, Yamashita H, Noma H, Mimura T, Yamashita T, Hori S. Increased levels of vascular endothelial growth factor and interleukin-6 in the aqueous humor of diabetics with macular edema. Am J Ophthalmol. 2002;133(1):70-7.

12. Nauck M, Karakiulakis G, Perruchoud AP, Papakonstantinou E, Roth M. Corticosteroids inhibit the expression of vascular endothelial growth factor gene in human vascular smooth muscle cells. Eur J Pharmacol. 1998;341(2-3):309-15.
13. Antcliff RJ, Spalton DJ, Stanford MR, Graham EM, Ffytche TJ, Marshall J. Intravitreal triamcinolone for uveitic cystoid macular edema: an optical coherence tomography study. Ophthalmology. 2001;108(4):765-72.

14. Martidis A, Duker JS, Greenberg PB, Rogers AH, Puliafito CA, Reichel E, et al. Intravitreal triamcinolone for refractory diabetic macular edema. Ophthalmology. 2002;109(5):920-7.

15. Moshfeghi DM, Kaiser PK, Scott I, Sears JE, Benz M, Sinesterra JP, et al. Acute endophthalmitis following intravitreal triamcinolone acetonide injection. Am J Ophthalmol. 2003;136(5):791-6. Comment in: Am J Ophthalmol. 2003;136(5):918-9. Am J Ophthalmol. 2004;137(6):1158-9; author reply 1160-1. Am J Ophthalmol. 2004;137(6):1159-60; author reply 1160-1. Am J Ophthalmol. 2004;137(6):1166; author reply 1167

16. McCuen BW, Bessler M, Tano Y, Chandler D, Machemer R. The lack of toxicity of intravitreally administered triamcinolone acetonide. Am J Ophthalmol. 1981;91(6):785-8.

17. Jonas JB, Hayler JK, Panda-Jonas S. Intravitreal injection of crystalline cortisone as adjunctive treatment of proliferative vitreoretinopathy. Br J Ophthalmol. 2000;84(9):1064-7.

18. Kivilcim M, Peyman GA, El-Dessouky ES, Kazi AA, Cheema R, Hegazy H. Retinal toxicity of triamcinolone acetonide in silicone-filled eyes. Ophthalmic Surg Lasers. 2000;31(6):474-8.

19. Vasconcelos-Santos DV, Nehemy PG, Schachat AP, Nehemy MB. Secondary ocular hypertension after intravitreal injection of $4 \mathrm{mg}$ of triamcinolone acetonide: incidence and risk factors. Retina. 2008;28(4):573-80.

20. Roth DB, Realini T, Feuer WJ, Radhakrishnan R, Gloth J, Heimmel MR, et al. Short-term complications of intravitreal injection of triamcinolone acetonide. Retina. 2008;28(1):66-70.

21. Rhee DJ, Peck RE, Belmont J, Martidis A, Liu M, Chang J, Fontanarosa J, et al. Intraocular pressure alterations following intravitreal triamcinolone acetonide. Br J Ophthalmol. 2006;90(8):999-1003.

22. Gillies MC, Sutter FK, Simposon JM, Larsson J, Ali H, Zhu M. Intravitreal triamcinolone for refractory diabetic macular edema: two year results of a double-masked, placebo-controlled, randomized clinical trial. Ophthalmology. 2006;113(9):1533-8.

23. Jonas JB, Kreissig I, Hugger P, Sauder G, Panda-Jonas S, Degenring R. Intravitreal triamcinolone acetonide for exsudative age related macular degeneration. Br J Ophthalmol. 2003;87(4):462-8.

24. Ozkiris A, Erkilic K. Complications of intravitreal injection of triamcinolone acetonide. Can J Ophthalmol. 2005;40(1):63-8.

25. Morlet N, Young SH. Prevention of intraocular pressure rise following intravitreal injection. Br J Ophthalmol. 1993;77(9):572-3.

26. Dwinger MC, Pieper-Bodeewes I, Eter N, Holz FG. [Variations in intraocular pressure (IOP) and necessity for paracentesis following intravitreal triamcinolone injection]. Klin Monatsbl Augenheilkd. 2005;222(8):638-42. 